\title{
Change in the ASF entry risk into Japan as a result of the COVID-19 pandemic
}

Katsuaki Sugiura $^{1}$, Katsumasa Kure ${ }^{2}$, Takuma Kato ${ }^{1}$, Fumiaki Kyutoku ${ }^{1}$, and Takeshi $\mathrm{Haga}^{3}$

${ }^{1}$ The University of Tokyo Graduate School of Agricultural and Life Sciences Faculty of Agriculture

${ }^{2}$ Value Farm Consulting

${ }^{3}$ University of Tokyo

June 25, 2020

\begin{abstract}
Using a model developed previously by the authors, a risk assessment was conducted to predict the change in the risk of ASF entering Japan as a result of the coronavirus pandemic in humans. The annual probability of ASF entering Japan was calculated to be $23 \%$ (90\% prediction interval: $0-91 \%$ ), 4.7\% (0-24\%) in February, 0.4\% (0-2.1\%) in March and 0.004\% (0-0.01\%) in April 2020 indicating a significant decline in the risk of ASF entry into Japan from China. The decline was attributed to a decline in the number of air travelers from China and amount of restaurant food.
\end{abstract}

Short Communication

Change in the ASF entry risk into Japan as a result of the COVID-19 pandemic Running Head: ASF RISK UNDER CORONAVIRUS PANDEMIC

Author's Name: K. Sugiura ${ }^{1}$, K. Kure ${ }^{2}$, T. Kato ${ }^{1}$, F. Kyutoku ${ }^{1}$ and T. Haga ${ }^{3}$

Affiliations:

1 Department of Global Agricultural Sciences, Graduate School of Agricultural and Life Sciences, the University of Tokyo.

Address: 1-1-1 Yayoi, Bunkyo-ku, Tokyo, 113-8657

2 Value Farm Consulting, 1704-3 Nishiooi, Tsukuba, Ibaraki 300-1260, Japan

3 Department of Veterinary Medical Sciences, Graduate School of Agricultural and Life Sciences, the University of Tokyo.

Address: 1-1-1 Yayoi, Bunkyo-ku, Tokyo, 113-8657

Correspondence:

Katsuaki Sugiura, Department of Global Agricultural Sciences, Graduate School of Agricultural and Life Sciences, The University of Tokyo.

Fax: 03-5841-5191

Tell:03-5841-5383 
Email: aksugiur@g.ecc.u-tokyo.ac.jp

\section{Summary}

Using a model developed previously by the authors, a risk assessment was conducted to predict the change in the risk of ASF entering Japan as a result of the coronavirus pandemic in humans. The annual probability of ASF entering Japan was calculated to be $23 \%$ (90\% prediction interval: 0-91\%), 4.7\% (0-24\%) in February, $0.4 \%(0-2.1 \%)$ in March and $0.004 \%(0-0.01 \%)$ in April 2020 indicating a significant decline in the risk of ASF entry into Japan from China. The decline was attributed to a decline in the number of air travelers from China and amount of restaurant food.

\section{KEY WORD:}

African swine fever (ASF), coronavirus (COVID-19), Japan, risk assessment

\section{Introduction}

African swine fever (ASF) is a highly contagious disease affecting pigs. It is caused by African swine fever virus (ASFV), which belongs to the genus Asfivirus of the Asfaviridae family (Alonso et al., 2018). ASF can spread through direct or indirect contact and causes high mortality. The ASFV persists for a long time in the environment and in a variety of pig products. Wild boar can harbor the virus and ASF may become endemic with or without an added transmission cycle through Ornithodoros ticks (Plowright et al., 1994). Traditionally ASF was confined to Africa, with occasional incursions into other regions until in 2007 when ASF was introduced into Georgia, a Caucasus country (Sanchez-Vizcaino et al.). From Georgia, the disease spread to other Caucasus and East European countries (European Food Safety Agency, 2010). Since ASF was reported in China for the first time in August 2018, it not only spread to within the country but to other Asian countries. Japanese pig population is petentially at risk of getting infected with ASFV as a result of being fed illegally imported pork products from ASF-affected countries (Sugiura et al., 2018). Based on this assumption, we have previously assessed the risk of ASF introduction into Japan through pork products illegally imported from China and fed to pigs as swill, using a stochastic model and the latest data available up to 2019 (Sugiura et al., 2020). As a result, the annual probability of ASFV entry into Japan via this pathway was predicted to be $20 \%$ (90\% prediction interval: 0-90\%) (Sugiura et al., 2020). However, under the various interventions taken to manage the human coronavirus outbreak that started in late 2019, there have been substantial changes in the value of some of the variables used in the model. These include the number of air travelers from China and the amount of food waste from restaurants. In this study we attempted to predict the change in the ASF entry risk into Japan after January 2020 due to the changes in these variable values under the coronavirus pandemic.

\section{Materials and Methods}

\section{ASF entry risk assessment model}

We used the risk assessment model that we previously developed (Sugiura et al., 2020), reparametrizing the variables that are deemed to be affected under the coronavirus pandemic, namely the number of air travelers from China and the amount of food waste from restaurants. We assumed that variables other than these two do not change even under the coronavirus pandemic. The number of infected pig farms in China remained more or less the same during the first four months of 2020 (OIE, 2020). The simulation was performed using @Risk Version 7.6 (Palisade, Ithaca, New York) within Microsoft@ Excel 2013, and was run with 10,000 iterations using Latin Hybercube sampling for each simulation.

\section{Number of air travelers from China in 2020}

As part of its response to managing the outbreak of the coronavirus (COVID-19), the Japanese government designated COVID-19 as a quaratinable infectious disease under Quarantine Act on 14 February 2020 and imposed travel restrictions against travelers entering Japan: travelers from specific areas of China and those 
who are suspected of being infected with COVID-19 were ordered to be quarantined. Also, since 31 January 2020 foreign nationals who were deemed to be COVID-19 patients were denied entry into Japan, pursuant to the Immigration Control and Refugee Recognition Act. As a result of these measures, the number of air travelers from China decreased dramatically since February 2020: the number of air travelers from China saw a change of $+22.6 \%,-87.9 \%,-98.5 \%$ and $-99.98 \%$ in during the first four months of 2020 respectively compared to the same months of the previous year (JINTO, 2020).

\section{Amount of food waste from restaurants under the coronavirus pandemic}

Since the last week of February when the Japanese government requested suspension of large-scale events, the number of people eating out in restaurants have started to decrease. This decrease was accelerated in late March when the Tokyo metropolitan governor requested residents to stay home during the weekends. On 7 April, the Japanese Prime Minister declared a state of emergency requesting residents in Tokyo and six other prefectures to stay home except for when needed and restaurants to shorten their opening hours. These measures decreased the restaurant's activity dramatically. According to the study by the Japan Food Service Association targeting its member restaurants, the sales of dining restaurants was $102.3 \%, 97.4 \%, 59.5 \%$ and $16.0 \%$ during the first four months of 2020 respectively compared to the same months of the previous year (Japan Food Service Association, 2020). We assumed that the food waste from restaurants decreased by these proportions during this period.

\section{Results and Discussion}

The annual probability of ASF entry into Japan in January 2020 was 23\% (90\% prediction interval: 0-91\%), slightly increased compared to the average annual probability in 2019 mainly due to the increase of air travelers from China. However, it decreased dramatically during the following three months: 4.7\% (0-24\%) in February, 0.4\% (0-2.1\%) in March and 0.004\% (0-0.01\%) in April 2020, due to the decrease both in the number of travelers from China and the amount of food waste from restaurants (Fig. 1).

These results indicate that there has been a dramatic decline in the ASF entry risk into Japan in early 2020, a reduction of 5000 fold. Although the model used in this study did not consider the entry risk relating to foreign workers, this risk is also predicted to be lowered as there were fewer foreign workers coming to work on farms in Japan under the travel restrictions (Nikkei Business News, 2020).

The ASF entry risk should be periodically monitored so that, depending on the predicted risk, Japanese pig farmers can be on alert to protect their farms from the introduction of ASF. The authors recommend that they utilize this low risk time to raise their level of biosecurity by, for example, contracting a veterinarian to develop an animal health plan and making a structural change in relation to pig houses, their layout and security fencing. This also applies to pig farms in other countries and regions where the ASF's entry risk was high until last year and declined in the COVID 19 pandemic.

\section{Acknowledgements}

This work was supported by the Ito Foundation (Ken-125, Fiscal Year 2020).

\section{Ethical statement}

This work includes no original research data and requires no ethical approval.

\section{Conflict of interest}

The authors declare no potential conflict of interests with respect to the research, authorship and/or publication of this article.

\section{References}

Alonso, C., Borca, M., Dixon, L., Revilla, Y., Rodriguez, F., Escribano, J.M., ICTV Report Consortium. (2018). ICTV Virus Taxonomy Profile:Asfarviridae. J.Gen. Virol. 99:613-614. 
European Food Safety Authority (EFSA) (2010). Scientific opinion on African swine fever. EFSA Journal, $8: 1556$.

JNTO (2020, May 25). Number of travelers visiting Japan (estimated number for April 2020). Retrieved fromhttps://www.jnto.go.jp/jpn/statistics/data_info_listing/pdf/200520_monthly.pdf

Japan Food Service Association (2020, May 2020). Report of restaurant market trend study for April 2020. Retrieved fromhttp://www.jfnet.or.jp/files/getujidata-2020-04.pdf

Nikkei Business News (2020, May 25). 15 March Issue - CronaShock - Embarrased foreign workers and start of labor shortage time. Retrieved fromhttps://business.nikkei.com/atcl/NBD/19/depth/00541/

OIE (2020, May 25). World Animal Health Information System (WAHIS) database. Retrieved fromhttps://www.oie.int/wahis_2/public/wahid.php/Countryinformation/Countryreports

Plowright, W., Thomson, G. R. \& Neser, J. A. (1994). African swine fever: Infectious diseases of livestock, with special reference to southern Africa (vol 1, 1st ed.) Cape Town, South Africa, Oxford University Press.

Sugiura K. \& Haga, T. (2018). A rapid risk assessment of African swine fever introduction and spread in Japan based on expert opinions. J Vet Med Sci. 80: 1743-1746.

Sugiura K., Lei Z., Holley C. \& Haga T. (2020). Assessing the risk of ASFV entry into Japan through pork products illegally brought in by air passengers from China and fed to pigs in Japan. PLoS One. 2020 May 5;15(5):e0232132. doi: 10.1371/journal.pone.0232132. eCollection 2020. PMID: 32369517

Sánchez-Vizcaíno, J.M., Mur, L, \& Martínez- López, B. (2012). African Swine Fever: An Epidemiological Update. Transbound. Emerg. Dis. 59:27-35.

\section{Figure legends}

Fig. 1

Evolution of annual risk of ASF entry into Japan during the first four months of 2020. For each box-whisker plot, the line in the box indicates the mean; the length of the box indicates the inter-quartile range; the whiskers indicate the 5 th percentile and the 95 th percentile respectively.

\section{Hosted file}

Figuress.docx available at https://authorea.com/users/336789/articles/462485-change-in-theasf-entry-risk-into-japan-as-a-result-of-the-covid-19-pandemic 